\title{
过渡金属催化肜类化合物的反应研究进展
}

\author{
由陇飞 梁浩关正辉* \\ (合成与天然功能分子化学教育部重点实验室 西北大学化学与材料科学学院 西安 710069)
}

\begin{abstract}
摘要 按照反应类型的不同，对近年来过渡金属催化肜类化合物反应的研究进展进行了综述，主要包括酮肜的还原酰 化反应，肟类化合物的催化偶联反应，胺化反应以及肜类化合物与炔烃的环化反应.

关键词 肟; 过渡金属; 还原酰化; 催化偶联; 胺化; 环化
\end{abstract}

\section{Progress in Transition Metal-Catalyzed Reactions of Oximes and Their Derivatives}

\author{
Ran, Longfei Liang, Hao Guan, Zhenghui* \\ (Key Laboratory of Synthetic and Natural Functional Molecule Chemistry of Ministry of Education, \\ Department of Chemistry \& Materials Science, Northwest University, Xi'an 710069)
}

\begin{abstract}
The recent progress in transition metal-catalyzed reactions of oximes and their derivatives is reviewed in this paper. The reactions mainly based on reductive acylation, catalyzed coupling, amination, and cyclization of oximes and their derivatives.

Keywords oximes; transition metal; reductive acylation; cross coupling; amination; cyclization
\end{abstract}

肜是一类非常重要的有机化合物, 其在醛酮的保护 与脱保护、贝克曼重排合成酰胺、醛肜脱水合成腈等方 面具有广泛的用途, 另外肟在合成手性胺、药物中间体 以及具有生物活性的各类杂环化合物等方面也具有巨 大的潜力. 因此, 用肜类化合物构筑结构复杂的碳-碳、 碳一杂原子键一直是化学工作者所关注的重要领域之一.

近几十年来, 人们在钯、铑、铜等过渡金属催化的 肜类化合物新反应研究方面, 已经取得了巨大的进展, 发现了许多有效的催化体系. 在此, 我们结合自己的研 究课题对该领域的一些研究进展进行了总结.

\section{1 酮肟的还原酰化反应}

烯酰胺及其衍生物广泛存在于天然产物及合成药 物之中. 它们是非常重要的有机合成中间体, 常作为原 料用于亲核加成、环加成、交叉偶联等反应中. 在立体 选择性构筑 $\mathrm{C}-\mathrm{C}, \mathrm{C}-\mathrm{N}$ 键, 特别是在不对称催化氢化 合成手性胺等领域具有重要的用途. 因此, 烯酰胺的合
成一直是化学工作者研究的一个热点.

1975 年, Barton 等 ${ }^{[1]}$ 报道了用酮肜和乙酸䣲为原料, 吡啶为溶剂合成烯酰胺的方法(Eq. 1). 该小组研究了不 同还原剂对反应的影响, 结果发现 $\mathrm{Ti}(\mathrm{OAc})_{3}$ 比 $\mathrm{Cr}(\mathrm{OAc})_{2}$ 具有更好的还原效果, 并随后又报道了用 $\mathrm{Fe}$ 粉作还原 剂的酮肜还原酰化反应 ${ }^{[2]}$.

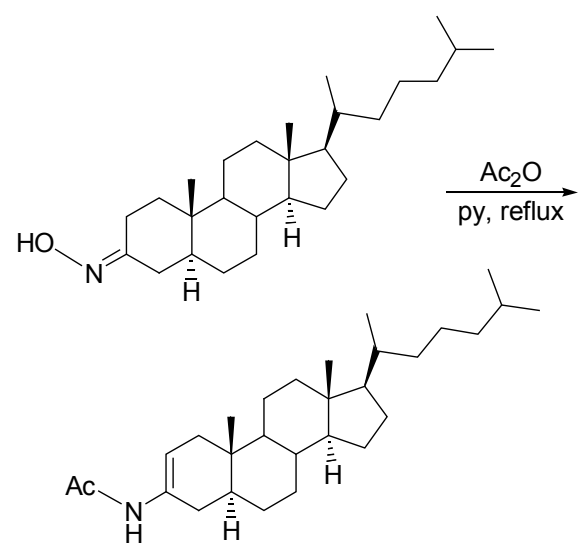

* E-mail: guanzhh@nwu.edu.cn

Received June 9, 2012; revised August 29, 2012; published online September 10, 2012.

Project supported by the National Natural Science Foundation of China (Nos. 21272183, 21002077), the Fund of New Scientific Stars of Shanxi Province (No. 2012KJXX-26) and the Fund of Education Department of Shaanxi Provincial Government (No. 12JK0611).

国家自然科学基金(Nos. 21272183，21002077)、陕西省科技厅 “陕西省青年科技新星” 基金(No. 2012KJXX-26)和陕西省教育厅基金(No. 12JK0611) 资助项目。 
1998 年, Burk 等 ${ }^{[3]}$ 研究发现在乙酸和甲苯作用下, 用 $\mathrm{Fe} / \mathrm{Ac}_{2} \mathrm{O}$ 也可以将肜转化成烯酰胺(Eq. 2). 此反应对 于环状或非环状酮肟都能得到较好的结果, 当 $\mathrm{R}^{1}$ 为非 氢原子取代基时, 所得产物为 $E / Z$ 型混合物 $(E: Z=3$ : 2). 同年, Zhang 等 ${ }^{[4]}$ 报道了以 $\mathrm{DMF}$ 作溶剂 $\mathrm{Fe} / \mathrm{Ac}_{2} \mathrm{O}$ 的酮 肜还原酰化体系, 获得了较好的结果. DMF 溶剂的使用 降低了反应温度, 提高了反应的转化率.

$$
\overbrace{\mathrm{R}^{1}}^{\mathrm{NOH}} \frac{\mathrm{Fe}, \mathrm{Ac}_{2} \mathrm{O}, \mathrm{HOAc}}{\text { toluene, } 75{ }^{\circ} \mathrm{C}}
$$

2008 年, Singh 等 ${ }^{[5]}$ 使用三烷基膦作为还原剂用于 酮肜的还原酰化反应, 获得了较好的结果(Scheme 1). 他们尝试了一系列有机膦化合物, 其中 1,2-双(二苯基 膦)乙烷(DPPE)、三乙基膦 $\left(\mathrm{Et}_{3} \mathrm{P}\right) 、$ 、丁基膦 $(n-\mathrm{Bu})_{3} \mathrm{P}$ 都 取得了很好的效果. 该方法操作简单, 所得产物易于分 离纯化, 适用于烯酰胺的大量制备, 但是由于含磷化合 物具有一定的毒性, 并且环境不友好, 所以一定程度上 限制了反应的应用范围.

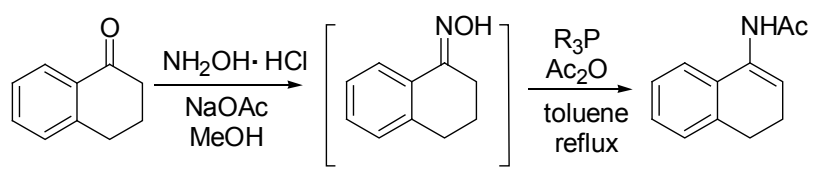

\section{Scheme 1}

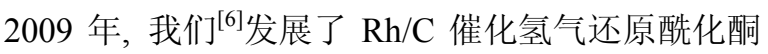
肜合成烯酰胺的新方法(Eq. 3). 该方法催化剂用量较少, 仅为底物的 $0.5 \mathrm{~mol} \%$, 产率高, 实验方法简单, 条件温

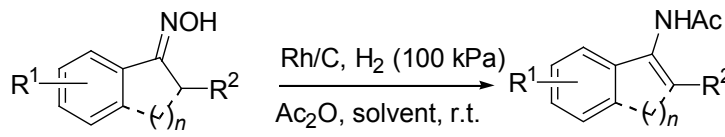<smiles>C=C(NC(C)=O)c1ccccc1</smiles>

$165 \%$<smiles>CC(=O)NC1=CCCc2ccccc21</smiles>

$285 \%$<smiles>CC(C)NC(=C1CCCCC1)c1ccccc1</smiles>

$370 \%$<smiles>C=C(N)c1ccc(C2CCCCC2)cc1</smiles>

$466 \%$<smiles>CNC1=CCc2ccccc21</smiles>

$588 \%$

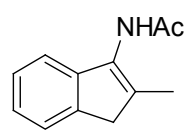

$682 \%$<smiles>CCCCCCCNC1=CCc2ccc(OC)cc21</smiles>

$782 \%$<smiles>CNC1=CCc2cc(OC)c(OC)cc21</smiles>

和, 反应在室温下即可进行, 特别是对于环状烯酰胺的 合成更为有效.

同年, Tang 等 ${ }^{[7]}$ 采用 $\mathrm{Fe}(\mathrm{OAc})_{2}$ 代替 $\mathrm{Fe}$ 粉作还原剂 进行肜的还原酰化, 得到了较好的结果(Eq. 4). 此方法 可以避免铁粉在醋酸溶剂中的放热现象，大大减少铁的 用量，反应条件温和，产物易分离纯化且收率高，是合 成烯酰胺的一条有效途径.

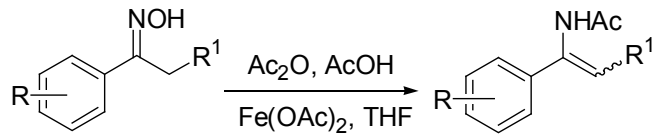

最近，我们 ${ }^{[8]}$ 发展了 $\mathrm{CuI}$ 催化的酮肜还原酰化制备 烯酰胺的新方法, 以 $\mathrm{NaHSO}_{3}$ 作还原剂, 在 1,2-二氯乙 烷溶剂中，合成了一系列不同结构的烯酰胺(Eq. 5). 与 其他方法相比, 具有原料易得, 官能团容忍度高, 操作 简便，成本低等优点，更为重要的是还原剂 $\mathrm{NaHSO}_{3}$ 的 运用，使该工艺无论在有机合成还是在工业应用方面都 有很好的前景.

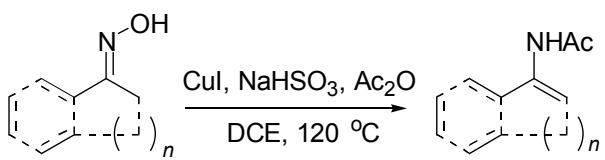<smiles>C=C(NC(C)=O)c1ccccc1</smiles>

$183 \%$<smiles>C=C(N)c1ccc(F)cc1</smiles>

$1177 \%$<smiles>C=C(NC(C)=O)c1ccc2c(c1)CCCC2</smiles>

$1495 \%$
$1071 \%$<smiles>C=C(NC(C)=O)c1ccccc1</smiles>

$1369 \%$<smiles>NC1=CCCc2ccccc21</smiles>

2 92\%

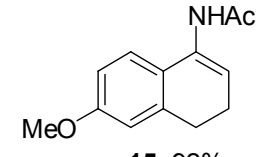

15 92\%

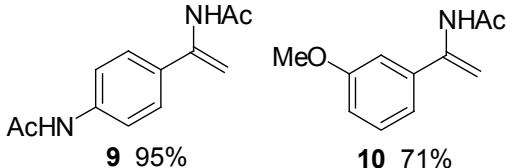<smiles>CCCNC1=CCc2ccccc21</smiles>

$574 \%$<smiles>CCNC1=CCc2cc(Cl)ccc21</smiles>

$1679 \%$

我们推测该反应的机理可能是通过以下两个主要 步骤完成的, 如 Scheme 2 所示: (1)酮肟在乙酸酐的作用 下生成肟酯中间体 $\mathbf{A}$; (2)肟酯 $\mathbf{A}$ 在 $\mathrm{Cu}(\mathrm{I})$ 作用下, 经过两 步电子转移得到氮负离子中间体 $\mathbf{C}$, 随后 $\mathbf{C}$ 被酸酤酰化 得酰亚胺 $\mathbf{D}$ ，异构化即得目标产物烯酰胺 $\mathbf{E}$. 


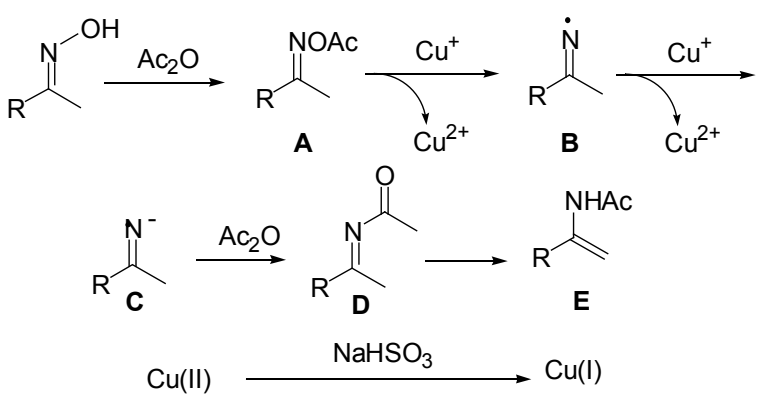

Scheme 2

\section{2 肟类化合物的催化偶联反应}

过渡金属催化的偶联反应是形成碳一碳键, 碳一杂原 子键最有效的方法之一, 目前已被广泛应用于有机合成 领域. 近年来, 过渡金属催化肜类化合物参与的偶联反 应逐渐被人们关注, 主要集中在寻找新的催化体系和配 体，探索反应机理和扩大反应的应用领域等方面.

1984 年, Cofffen 等 ${ }^{[9]}$ 在 $\left(\mathrm{PPh}_{3}\right)_{2} \mathrm{PdCl}_{2}$ 和 $\mathrm{CuI}$ 催化下, 以化合物 17 作底物, 室温下即可发生分子内的 $\mathrm{C}-\mathrm{O}$ 键 偶联反应，得到苯并异恶唑衍生物 18, 产率达到 80\%, 但因缺乏底物通用性，该方法并没有被广泛应用(Eq. 6).
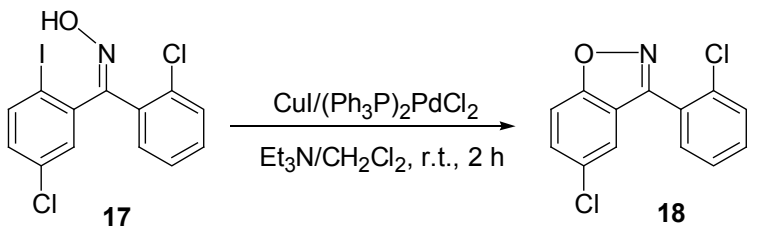

在此基础上, Maitra 和 Wailes 等 ${ }^{[10]}$ 使用 1,10 -菲啰啉 作为配体, $\mathrm{CuI}$ 为催化剂, 甲苯或 DMSO 作溶剂, $\mathrm{Cs}_{2} \mathrm{CO}_{3}$ 为碱的条件下实现了芳基酮肜及醛肜与溴代、碘代芳香 烃分子间的 $\mathrm{C}-\mathrm{O}$ 键交叉偶联反应，用以合成肜醚衍生 物(Eq. 7). 在该反应体系中, 酒石酸钾钠作为一种添加 剂, 在反应过程中起到了重要作用, 这是因为它可以与 反应过程中生成的 $\mathrm{Cu}(\mathrm{II})$ 形成螯合物，有效地抑制了酮 肜的脱肜分解副反应.

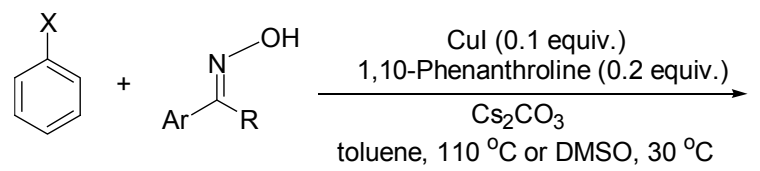

$\mathrm{X}=\mathrm{Br}, \mathrm{I}$<smiles>[R]/C([Al])=N/Oc1ccccc1</smiles>

$\mathrm{R}=$ Aromatic, Alkyl groups, $\mathrm{H}$

2010 年, Tois 等 ${ }^{[11]}$ 报道了应用 N,N 型配体，使用 $\mathrm{CuI}$ 作为催化剂, 以 $\mathrm{THF}$ 作溶剂, $t-\mathrm{BuONa}$ 为碱在室温条
件下催化了分子内的 $\mathrm{C}-\mathrm{O}$ 键偶联反应，成功合成了一 系列异噁唑类化合物 20 (Eq. 8). 他们对多种配体进行 了考察，发现 N,N 型配体 1,10-菲啰啉 L1，四甲基乙二 胺(TMEDA) L2, $N, N^{\prime}$-二甲基乙二胺(DMEDA) $\mathbf{L} 3$ 对于 该反应都表现出了很好的活性，而甘氨酸配体 L4 的活 性却很差.

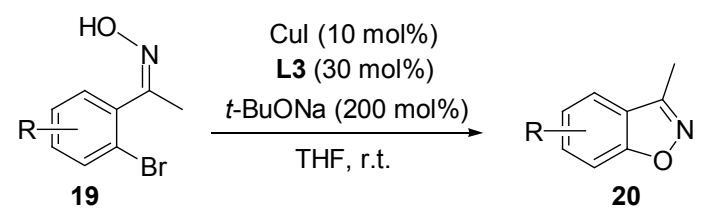<smiles>c1cnc2c(c1)ccc1cccnc12</smiles>

L1<smiles>CN(C)CCN(C)C</smiles>

L2<smiles>CNCCNC</smiles>

L3<smiles>NCC(=O)O</smiles>

同年, Buchwald 等 ${ }^{[12]}$ 报道了 $(\text { allyPdCl })_{2}$ 作为催化剂, 二芳基膦为配体，甲苯为溶剂， $\mathrm{CsCO}_{3}$ 为碱， $65{ }^{\circ} \mathrm{C}$ 条件 下芳香卤代物与乙酰羟肜酸乙酯 $\mathbf{2 1}$ 的 $\mathrm{C}-\mathrm{O}$ 键偶联反 应，反应产率为 70\% 95\% (Eq. 9). 他们发现加入催化 量的二芳基膦配体 L5, L6 是该反应成功的关键，主要 原因在于它可以有效地促进钯的还原消除.
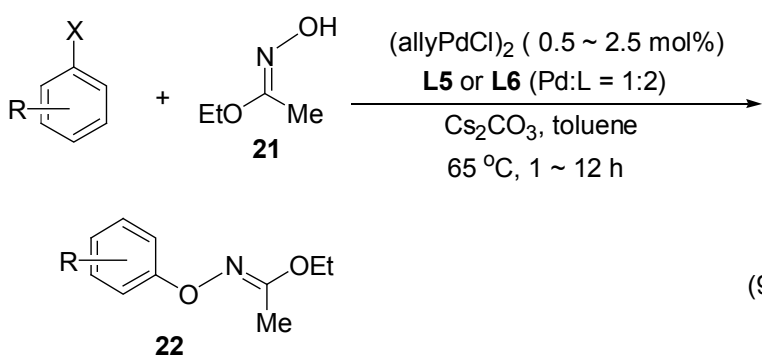

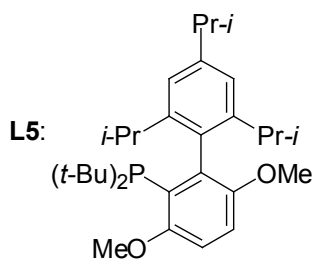

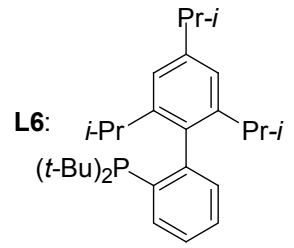

此外，过渡金属催化的肜类化合物与芳基硼酸的偶 联反应由于反应条件温和、有机硼试剂低毒以及稳定性 好，底物适用范围广，产物易于分离等特点，已被应用 于碳-碳、碳一氮键的构筑.

2007 年, Liebeskind 等 ${ }^{[13]}$ 报道了一种条件温和并且 高效的铜催化 $\mathrm{C}-\mathrm{N}$ 键偶联反应(Eq. 10). 该反应使用 $\mathrm{CuTC}$ 或 $\mathrm{Cu}(\mathrm{OAc})_{2}$ 作为催化剂, 在无外加氧化剂和碱的 条件下, 肟酯与芳基硼酸(或有机锡化合物)偶联合成亚 胺.

研究者在拓展底物时发现, 当芳基硼酸作为偶联试 剂时，五氟苯甲酰肜酯相对于乙酸肟酯具有更好的反应 
效果. 然而, 当有机锡化合物作为偶联试剂时, 乙酸肜 酯化合物则反应的更好. 值得一提的是, $E / Z$ 型的肟酯 化合物均能得到相同比例的目标产物, 表明该反应不会 受到立体化学的影响.

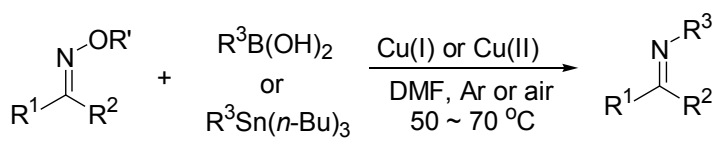

$2352 \% \sim 98 \%$

$\mathrm{R}^{\prime}=\mathrm{Ac}, \mathrm{COC}_{6} \mathrm{~F}_{5}$

$\mathrm{R}^{1}, \mathrm{R}^{2}=$ aryl , heteroaromatic, alkyl

$\mathrm{R}^{3}=$ aryl, alkenyl for B; aryl, heteroaryl, and alkenyl for Sn

此外, 作者对反应的机理也给出了合理的解释: 首 先 $\mathrm{Cu}(\mathrm{I})$ 催化剂与肜酯化合物中的 $\mathrm{N}-\mathrm{O}$ 键氧化加成, 随 后参与反应的芳基硼酸或有机锡化合物发生转金属化 反应，最后还原消除即得最终产物(Scheme 3).

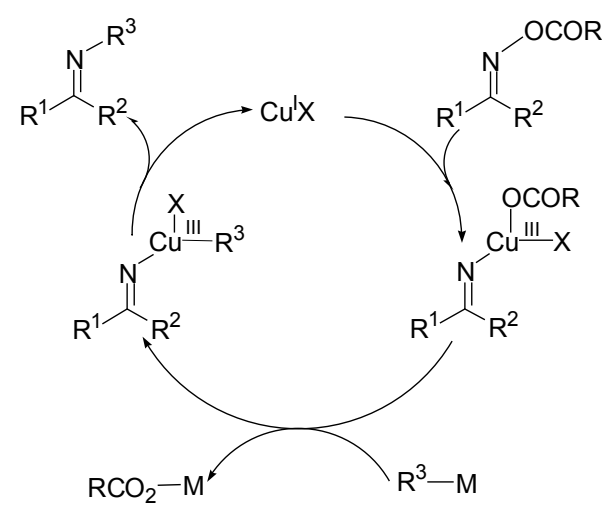

Scheme 2

2008 年, Liebeskind 等 ${ }^{[14]}$ 又报道了采用 DMF 作溶 剂, $\mathrm{Cu}(\mathrm{OAc})_{2}$ 作催化剂, 在 $4 \AA$ 分子篮共同作用下 $\alpha, \beta$ 不饱和肟酯和烯基硼酸的 $\mathrm{C}-\mathrm{N}$ 键偶联反应, 该方法操 作简单，条件温和，产物 26 收率从中等到优良 $(43 \%$ $91 \%$ ), 为合成多取代的吡啶衍生物提供了一种有效的 方法(Eq. 11). 他们研究发现, 该反应对硝基、酯基、腈 基、氯和澳等取代基都具有很好的官能团容忍性, 硒代 化合物也能够得到对应目标产物, $4 \AA$ 分子篮的作用是 防止中间体氮杂三烯的水解.

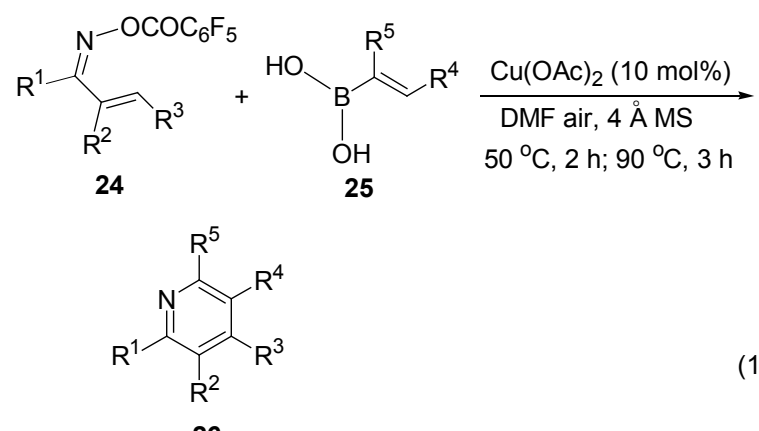

反应可能的机理如 Scheme 4 所示: 首先 $\alpha, \beta$-不饱和 肟酯与烯基硼酸在铜盐的催化下发生 $\mathrm{C}-\mathrm{N}$ 键偶联，随 后偶联产物发生电环化反应，再经过空气氧化即得目标 产物.
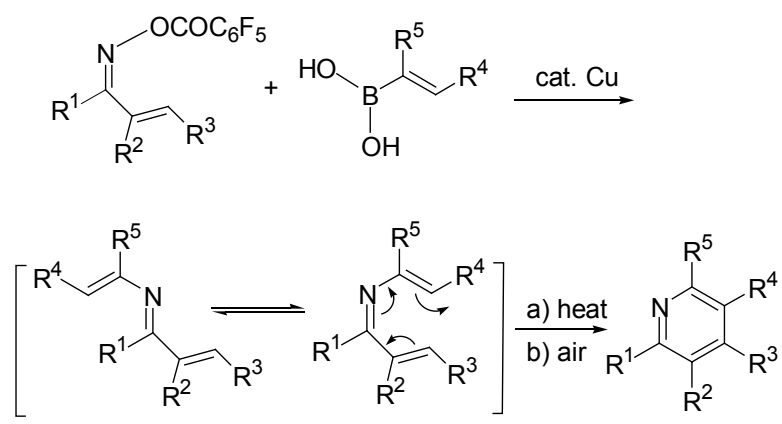

Scheme 4

2009 年, Huang ${ }^{[15]}$ 和 Meyer 等 ${ }^{[16]}$ 几乎同时报道了使 用 $\mathrm{Cu}(\mathrm{OAc})_{2}$ 作催化剂, 以吡啶为碱, 1,2 -二氯乙烷作溶 剂, 在室温条件下芳香肟和苯基硼酸的 $\mathrm{C}-\mathrm{O}$ 键偶联反 应(Eqs. 12, 13).

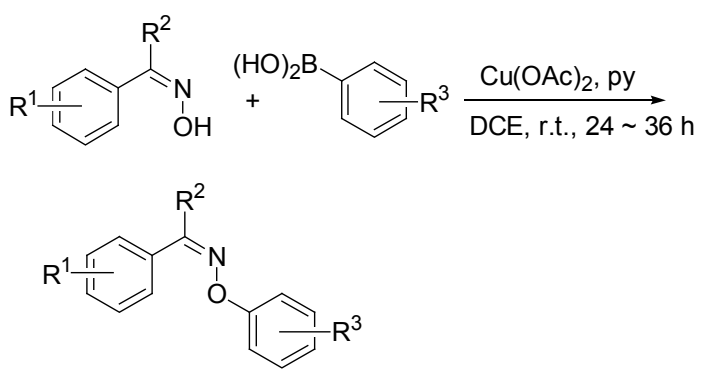

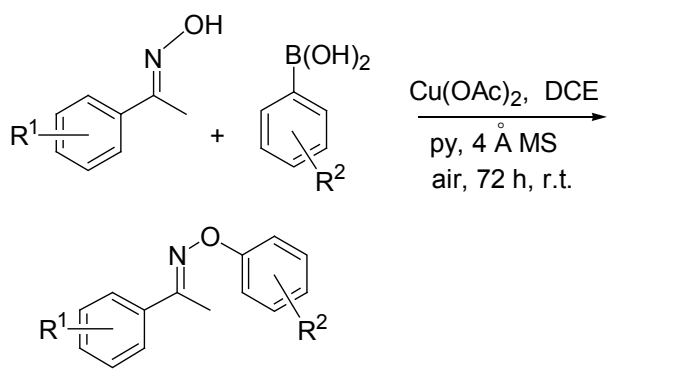

2010 年, $\mathrm{Ca}$ 等 ${ }^{[17]}$ 将一种新型固载 $\mathrm{Cu}$ 的聚合物作为 催化剂用于苯基硼酸和肜的 $\mathrm{C}-\mathrm{O}$ 键偶联反应，取得了 很好的效果(Eq. 14). 由于这种聚合物固载的金属催化 剂有着很高的催化活性和选择性，并可回收循环利用， 因此在有机合成化学中具有一定的应用前景.

虽然关于肜类化合物的催化偶联反应研究已经取 得了一定的进展，但寻找高效、多功能和更简单的催化 体系仍然是本领域的研究热点之一. 最近, 我们 ${ }^{[18]}$ 报道 了 $\mathrm{CuBr}$ 催化肜酯与醛的偶联反应合成吡啶类化合物的 新方法(Eq. 15).

我们首先研究了以 $\mathrm{CuI}$ 作催化剂, DMSO 作溶剂的 条件下苯乙酮肜酯与苯甲醛的偶联反应情况，令人满意 
<smiles>[R]C([R16])=NOc1cccc([R])c1</smiles><smiles>[CH]1CC[SiH2]1</smiles>

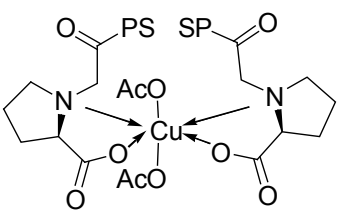<smiles>[R1]c1ccc2c(c1)C(=NOC(C)=O)C[Y8]2=O</smiles>
$n=1,2$

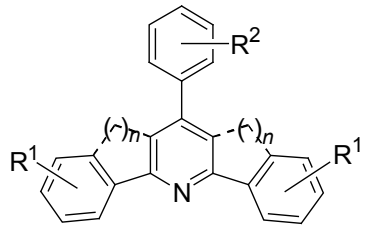

地得到了对称的三取代吡啶化合物, 产率达 $30 \%$. 在此 基础上, 对反应条件进行了系统研究(结果见表 1 ), 发现 DMSO 是理想溶剂, 在反应温度 $120{ }^{\circ} \mathrm{C}$, 催化剂 $\mathrm{CuBr}$ 用量为 $10 \mathrm{~mol} \%$ 的条件下, 催化反应效果最佳. 在该催 化体系中加入 $\mathrm{NaHSO}_{3}$ 能够有效地抑制肜酯的分解, 提 高反应效率.

表 1 不同催化剂和溶剂对肜酯与醛偶联反应的影响 ${ }^{\mathrm{a}}$

Table 1 Influences of different catalysts and solvents on the coupling of oxime acetate with aldehyde

\begin{tabular}{ccccc}
\hline Entry & Catalyst & Additive & Solvent & Yield/\% \\
\hline 1 & $\mathrm{CuI}$ & - & DMSO & 30 \\
2 & $\mathrm{CuI}$ & $\mathrm{NaHSO}_{3}$ & DMSO & 79 \\
3 & $\mathrm{CuBr}$ & $\mathrm{NaHSO}_{3}$ & DMSO & 89 \\
4 & $\mathrm{CuCl}$ & $\mathrm{NaHSO}_{3}$ & DMSO & 77 \\
5 & $\mathrm{Cu} 2$ & $\mathrm{NaHSO}_{3}$ & DMSO & 61 \\
6 & $\mathrm{Cu}(\mathrm{OAc})_{2}$ & $\mathrm{NaHSO}_{3}$ & DMSO & 60 \\
7 & $\mathrm{CuBr}$ & $\mathrm{NaHSO}_{3}$ & NMP & 78 \\
8 & $\mathrm{CuBr}$ & $\mathrm{NaHSO}_{3}$ & $1,4-$ Dioxane & 42 \\
9 & $\mathrm{CuBr}$ & $\mathrm{NaHSO}_{3}$ & Toluene & 35 \\
10 & $\mathrm{CuBr}$ & $\mathrm{Na}_{2} \mathrm{SO}_{3}$ & DMSO & 32 \\
\hline
\end{tabular}

${ }^{a}$ Reaction conditions: acetophenoe oxime acetate, $0.9 \mathrm{mmol}$; benzaldehyde, $0.3 \mathrm{mmol}$; catalyst, $10 \mathrm{~mol} \%$; additive, $0.9 \mathrm{mmol}$; solvent, $5 \mathrm{~mL}$. Ar at $120{ }^{\circ} \mathrm{C}$ for $2.5 \mathrm{~h}$.

实验表明：该催化体系具有广泛的底物适应性，含 有不同官能团的底物都能得到很好的结果. 富电子芳环 较缺电子芳环的肜酯化合物具有更高的反应活性，相 反，对于芳环上带有吸电子基团的醛类化合物则表现出 了更好的反应效果, 并且发现位阻效应对反应的影响甚 小. 值得注意的是, 在该反应条件下，两种不同的肜酯 与醛也可以进行交叉偶联, 三种吡啶化合物的总产率高
达 $82 \% \sim 92 \%$, 其中非对称的吡啶化合物为主要产物, 这为合成非对称的三取代吡啶化合物提供了一种新方 法(Eq. 16).

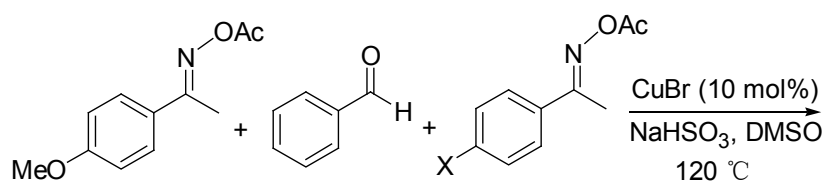<smiles>[X]c1ccc(-c2cc(-c3ccccc3)cc(-c3ccc(OC)cc3)n2)cc1</smiles><smiles>[X]c1ccc(-c2cc(-c3ccccc3)cc(-c3ccc([X])cc3)n2)cc1</smiles>

根据实验观察和相关文献报道, 我们提出了如 Scheme 5 所示可能的机理.
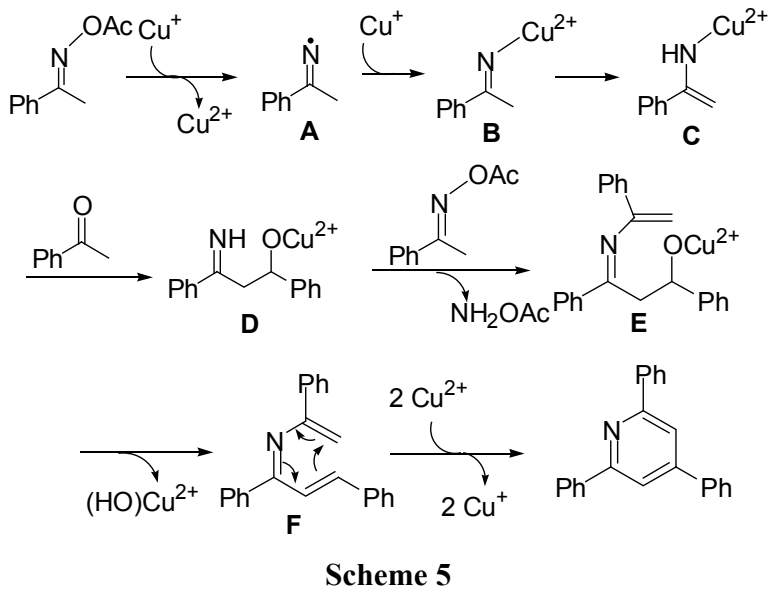

\section{3 肜类化合物的胺化反应}

1999 年, Narasaka 等 ${ }^{[19]}$ 用 $\mathrm{Pd}\left(\mathrm{PPh}_{3}\right)_{4} / \mathrm{Et}_{3} \mathrm{~N}$ 体系, DMF 作溶剂, 在 $80{ }^{\circ} \mathrm{C}$ 的条件下催化 $\gamma-\delta$ 不饱和肜酯化合物 发生分子内的胺化反应(即 Heck 胺化反应)，顺利合成了 吡咯类化合物 27. 随后，他们 ${ }^{[20]}$ 还将该反应运用到异喹 啉 28, 吡啶 29 等含氮杂环化合物的合成, 也都取得了 很好的效果(Scheme 6).

2005 年, Abell 等 ${ }^{[21]}$ 也用该催化体系成功合成了咪 唑类化合物 31 (Eq. 17). 
$\overbrace{\mathrm{DMF}, 80^{\circ} \mathrm{C}, 1 \mathrm{~h}}^{\stackrel{\mathrm{Pd}\left(\mathrm{PPh}_{3}\right)_{4}, \mathrm{Et}_{3} \mathrm{~N}}{\mathrm{CH}_{2} \mathrm{Cl}_{2} \text {, r.t., } 0.5 \mathrm{~h}}} \stackrel{\mathrm{Me}_{3} \mathrm{SiCl}}{\mathrm{OCOC}_{6} \mathrm{~F}_{5}}$

$2785 \%$

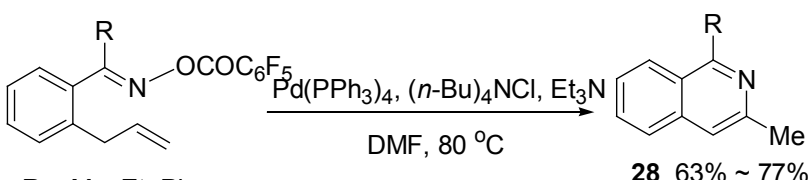

$\mathrm{R}=\mathrm{Me}, \mathrm{Et}, \mathrm{Ph}$

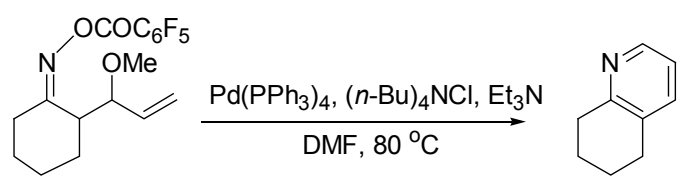

$2962 \%$

Scheme 6

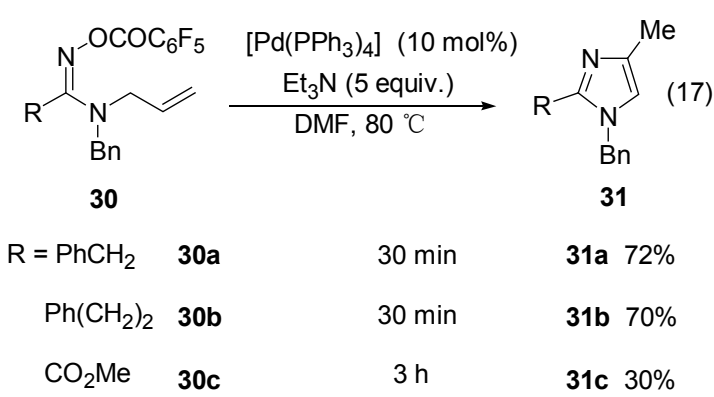

随后, Kamijo 等 ${ }^{[22]}$ 对该类反应的机理给出了较为合 理的解释, 如 Scheme 7 所示: 首先加入的 $\operatorname{Pd}(0)$ 催化剂 与㜆肜 30 中的 $\mathrm{N}-\mathrm{O}$ 键发生氧化加成生成 $\mathrm{Pd}(\mathrm{II})$ 中间体 $\mathbf{A}$, 然后分子内迁移插入到碳碳双键生成中间体 $\mathbf{B}$, 接 着 $\beta-\mathrm{H}$ 消除得到二氢咪唑 $\mathbf{C}$, 最后异构化生成产物 $\mathbf{3 1}$, 反应过程中生成的 $\mathrm{Pd}-\mathrm{H}$ 物种在 $\mathrm{Et}_{3} \mathrm{~N}$ 作用下, 重新生成 具有催化活性的 $\operatorname{Pd}(0)$ 络合物实现催化剂的循环.

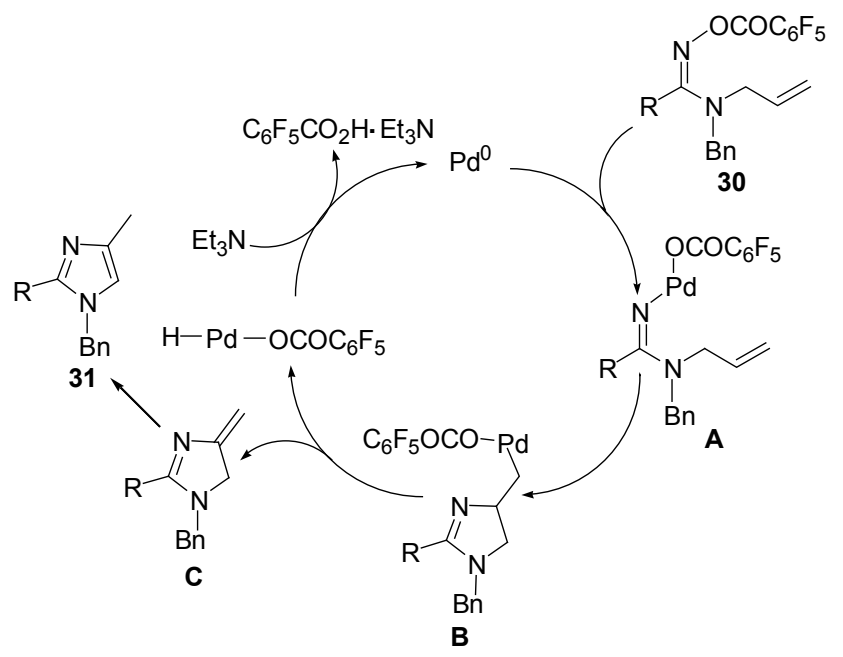

Scheme 7

尽管传统的钯催化 Heck 胺化反应在合成各类含氮
杂环化合物方面起着重要的作用, 但这种方法主要适用 于 $\gamma-\delta$ 不饱和肟酯类化合物的分子内胺化, 所以在一定 程度上限制了该类反应在有机合成中的应用 ${ }^{[23]} .2010$

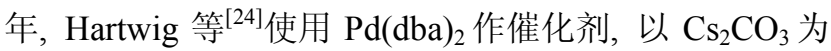
碱，甲苯作溶剂， $150{ }^{\circ} \mathrm{C}$ 温度下实现了肜酯类化合物分 子内芳环 $\mathrm{C}-\mathrm{H}$ 键直接胺化合成取代吲哚(Eq. 18). 该反 应无需外部氧化剂, 克服了传统方法利用芳香卤代物制 备芳胺类化合物所带来的环境污染, 为研究肜酯类化合 物的催化胺化反应提供了一种新的策略.

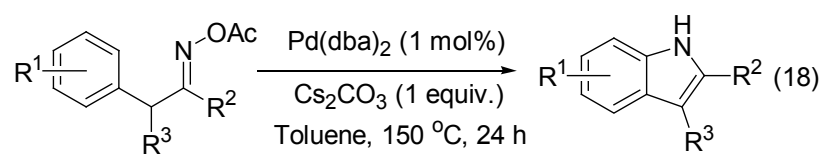

Hartwig 等对该反应的机理也进行了深入研究，并 给出了合理的机理解释 (Scheme 8). 首先催化剂 $\operatorname{Pd}(0)$ 与 肟酯化合物 32 中的 $\mathrm{N}-\mathrm{O}$ 键发生氧化加成, 生成中间体 I，然后异构化得中间体 II，中间体 II 发生邻位 C- $\mathrm{H}$ 键 活化得到中间体 III，最后通过还原消除生成目标产物 和 $\operatorname{Pd}(0)$ 催化剂, 或者是中间体 I 先发生 $\mathrm{C}-\mathrm{H}$ 活化最后 再异构化生成吲哚产物 33.

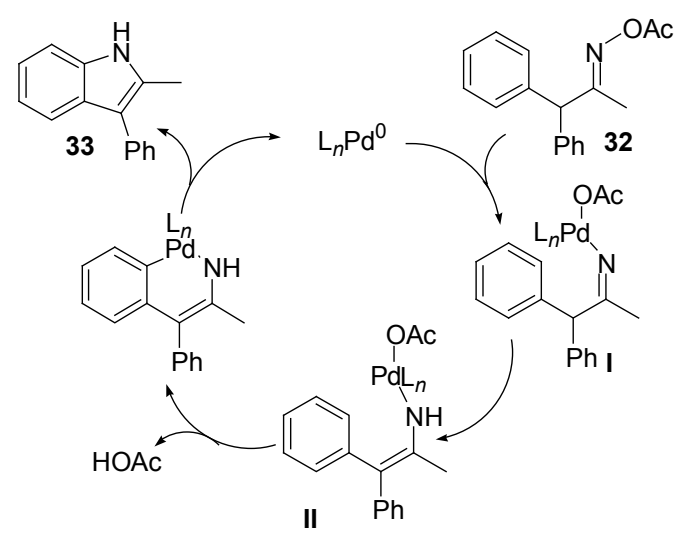

Scheme 8

值得注意的是，他们用 $\mathrm{Pd}\left(\mathrm{PCy}_{3}\right)_{2}$ 代替 $\mathrm{Pd}(\mathrm{dba})_{2}$ 作催 化剂与肜酯化合物 34 进行反应, 首次分离得到了 $\mathrm{Pd}(\mathrm{II})$ 化合物 35, 并用 X 射线衍射方法对 35 的结构进行了证 实. 在适当的反应条件 $\left[150{ }^{\circ} \mathrm{C}\right.$, 甲苯作溶剂, $\mathrm{Cs}_{2} \mathrm{CO}_{3}$ (1.0 equiv.)]下，化合物 35 可得到 31\%的目标化合物 36, 为该类反应的机理研究提供了重要线索 (Scheme 9).

几乎同时, Chiba 等 ${ }^{[25]}$ 报道了使用 $\mathrm{PdCl}_{2}(\mathrm{MeCN})_{2} /$ $\mathrm{MgO}$ 催化体系, 1,2 -二氯乙烷作溶剂, $80{ }^{\circ} \mathrm{C}$ 条件下催化 肜酯类化合物 37 经过分子内芳环 $\mathrm{C}-\mathrm{H}$ 键的直接胺化 合成取代吲哚 38 的反应(Eq. 19).

为了探索反应的机理，他们选用気标记的肪酯化合 物 39 为底物, 结果发现该反应的动力学同位素效应值 为 1 , 这说明该胺化反应经历的是一个亲电芳香取代过 


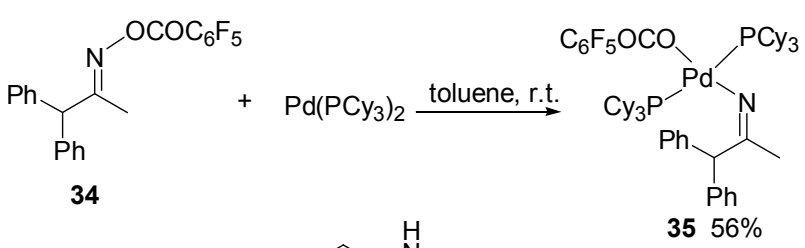

$\frac{\mathrm{Cs}_{2} \mathrm{CO}_{3}(1 \text { equiv. })}{\text { toluene, } 150^{\circ} \mathrm{C}, 2 \mathrm{~h}}$

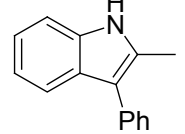

36 31\%

Scheme 9

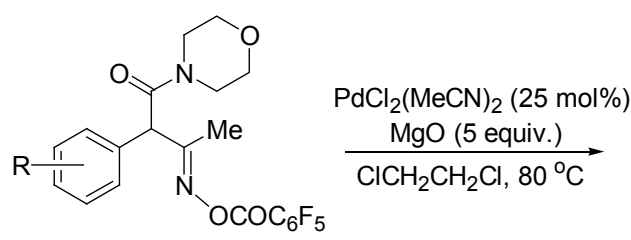

37<smiles>[R]c1ccc2[nH]c(C)c(C(=O)N3CCOCC3)c2c1</smiles>

38<smiles>[R]C(NOC(=O)OC)C([R])C1C=CCCC1C</smiles>

$\mathrm{R}=$ alkyl, aryl,cyclopropyl<smiles>[R]C1=NC2C=CCCC2C1[R]</smiles>

$4020 \% \sim 93 \%$<smiles>C1=C[C@H]2CC(c3ccccc3)=N[C@H]2C1</smiles>

$4182 \%$<smiles>Brc1ccc(C2=N[C@H]3C=CCC[C@H]3C2)cc1</smiles>

$4320 \%$
$\left[\mathrm{Pd}_{2}(\mathrm{dba})_{3}\right](2.5 \mathrm{~mol} \%)$

$\frac{\mathrm{P}\left(3,5-\left(\mathrm{CF}_{3}\right)_{2} \mathrm{C}_{6} \mathrm{H}_{3}\right)_{3} \quad(10 \mathrm{~mol} \%)}{\operatorname{DMF}(0.1 \mathrm{~mol} / \mathrm{L}), \mathrm{Et}_{3} \mathrm{~N}(4 \text { equiv. })}$ $60 \sim 120^{\circ} \mathrm{C}$
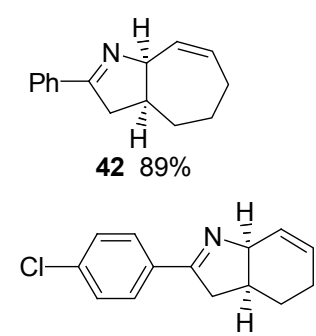

$4463 \%$

45 与炔的反应, 合成了取代吡啶类化合物 46, 收率可达 92\% (Eq. 22). 该方法也可以应用于异喹啉类化合物的 合成.

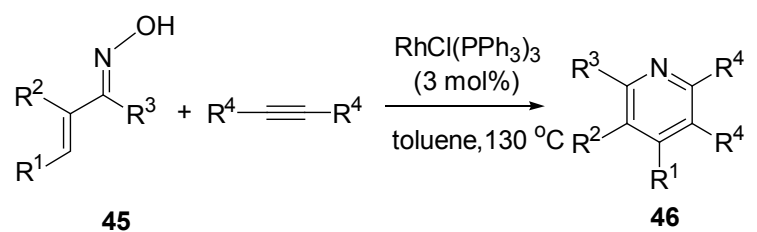

此外, Cheng 等还深入研究了肜 $\mathbf{4 7}$ 和 $\mathbf{4 8}$ 与不对称 炔烃进行加成环化反应时，这一催化体系对反应区域选 择性的影响(Scheme 10).<smiles>[2H]c1cccc2[nH]c(C)c(C(=O)N(C)C)c12</smiles>
种高效的配体被用于温和条件下的钯催化 NarasakaHeck 环化反应(Eq. 21). 实验结果发现, 不同类型的膦 配体如 Xantphos, $\mathrm{P}\left(p-\mathrm{FC}_{6} \mathrm{H}_{4}\right)_{3}, \mathrm{P}\left(p-\mathrm{CF}_{3} \mathrm{C}_{6} \mathrm{H}_{4}\right)_{3}$ 等在 $\mathrm{Pd}_{2}(\mathrm{dba})_{3}$ 的作用下对反应都有影响，当采用 $\mathrm{P}\left[3,5-\left(\mathrm{CF}_{3}\right)_{2} \mathrm{C}_{6} \mathrm{H}_{3}\right]_{3}$ 作为配体时效果最好, 目标产物 40 收 率可达 $93 \%$. 有趣的是, 含有环戍烯、环庚烯的底物也 能完成反应, 而且都得到了满意的结果; 含有氯、溴取 代基相对于无取代基的反应来说，所需温度较高，反应 时间较长，产率偏低.

\section{4 肜类化合物与炔的反应}

近几年来, 研究肜类化合物与炔烃在过渡金属催化 下的偶联反应也倍受关注. 2007 年, Cheng 等 ${ }^{[27]}$ 报道了 $\mathrm{RhCl}\left(\mathrm{PPh}_{3}\right)_{3}$ 作催化剂, 以甲苯作溶剂, $\alpha, \beta$-不饱和䣶肪<smiles>[R]C#C[C-]C(C)=NO</smiles>

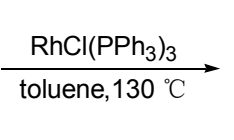<smiles>[R]c1ncc2c(c1-c1ccccc1)CCC2</smiles>
$\mathrm{R}^{5}=\mathrm{SiMe}_{3} \quad 80 \%$ $\mathrm{R}^{5}=\mathrm{H} 51 \%$<smiles>[R7]#C[C-](C)/C(=N\O)C1=CCCCC1</smiles>
$\underset{\text { toluene, } 130^{\circ} \mathrm{C}}{\mathrm{RhCl}\left(\mathrm{PPh}_{3}\right)_{3}}$<smiles>[R]c1c(-c2ccccc2)nc(C)c2c1CCCC2</smiles>

$\mathrm{R}^{5}=\mathrm{Me} \quad 48 \%$ $58.5 \%$<smiles>[R]c1nc(C)c2c(c1-c1ccccc1)CCCC2</smiles>

$\mathrm{R}^{5}=\mathrm{Et}$

Scheme 10

随后, Cheng 等 ${ }^{[28]}$ 进一步利用 $\mathrm{RhCl}\left(\mathrm{PPh}_{3}\right)_{3}$ 作催化剂 研究了不同类型酮肟化合物 $(49 a, 49 b, 49 c)$ 和炔的反应, 合成了异喹啉和四氢喹啉类衍生物 50 (Scheme 11). 该 体系对含有不同取代基团的肪类化合物都表现出了良 
好的适应性, 反应的产率都较好.

对于此反应, 他们提出了一个可能的机理, 如 Scheme 12 所示: 首先, 苯乙酮䏡 49a 中的 $\mathrm{N}$ 原子与 $\mathrm{Rh}(\mathrm{I})$ 配位，接着发生邻位 $\mathrm{C}-\mathrm{H}$ 活化生成中间体 $\mathbf{B}$, 中间体 $\mathbf{B}$ 脱去一个 $\mathrm{PPh}_{3}$ 配体后与炔烃配位, 再经过炔烃的顺式 插入, 得到中间体 $\mathbf{C}$ 或 $\mathbf{C}^{\prime}$, 随后还原消除得加成产物 $\mathbf{D}$, 最后发生电环反应, 脱一分子水即得目标产物 50a.

2011 年, Zhao, $\mathrm{Jia}$ 和 $\mathrm{Li}$ 等 ${ }^{[29]}$ 在 $\mathrm{CH}_{3} \mathrm{OH}$ 溶剂中, 采 用 $\left[\mathrm{RhCp}^{*} \mathrm{Cl}_{2}\right]_{2}$ 作催化剂, $\mathrm{CsOAc}$ 作添加物, 在 $60{ }^{\circ} \mathrm{C}$ 条 件下通过肜与炔烃的交叉偶联反应制得了异喹啉化合 物(Eq. 23). 该方法只需要 $1 \mathrm{~mol} \%$ 的催化剂, 产率就高 达 $95 \%$, 而且无需隔绝空气与水, 在温和条件下便能顺 利进行.

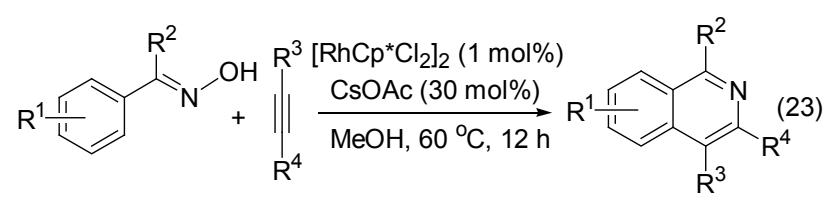

此外, 作者对反应的机理也进行了深入研究, 先是
提出了三种可能的机理(Scheme 13), 并逐一通过实验 对其进行了验证, 最终认为 Pathway c 所描述的机理最 为可能. 首先, $\mathrm{Rh}(\mathrm{III})$ 化合物与苯乙酮肜(51)发生亲电金 属化形成物种 I, 接下来与炔配位形成中间体 II，随后 在酸作用下质子化脱水生成铑 $(\mathrm{V})$ 物种 III, 经还原消除 即得目标产物, 同时释放出 $\mathrm{RhX}_{3}$, 从而完成催化循环.

最近, Ellman 等 ${ }^{[30]}$ 又报道了 $\alpha, \beta$-不饱和酮肜与末端 炔烃在 $\left[\mathrm{RhCl}(\mathrm{coe})_{2}\right]_{2} / \mathrm{P}(\mathrm{O}-i-\mathrm{Pr})_{3}$ 催化下的偶联环化反应, 一锅法顺利合成了多取代吡定化合物，产率从中等到优 良, 并且表现出了较高的立体选择性(Eq. 24). 研究者发 现, 在该反应中三异丙氧基膦 $\left(\mathrm{P}(\mathrm{O}-i-\mathrm{Pr})_{3}\right)$ 作为一种简单 廉价的有机磷配体可以有效抑制末端炔的自偶联.
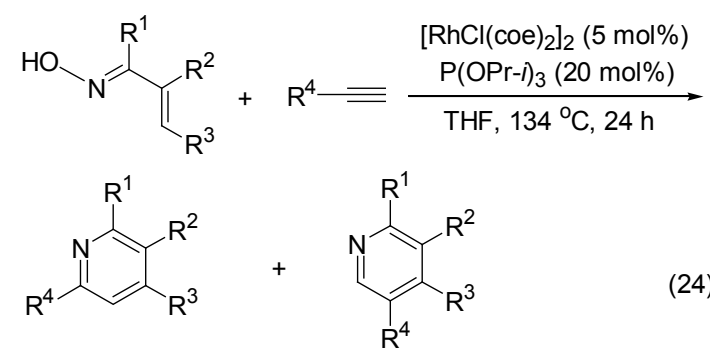<smiles>[R]c1ncc2ccccc2c1[R]</smiles><smiles>CC(=NO)c1ccccc1</smiles>

$50 a$
$\mathrm{RhCl}\left(\mathrm{PPh}_{3}\right)_{3}(3 \mathrm{~mol} \%)$

toluene, $130{ }^{\circ} \mathrm{C}, 12 \mathrm{~h}$

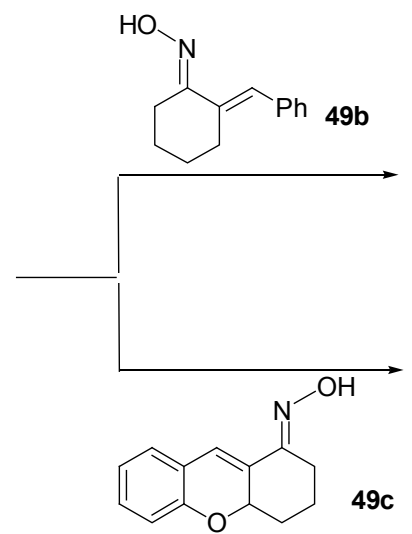<smiles>[R]c1nc2c(c([R])c1[R1])CCCC2</smiles><smiles>[R]c1nc2c3c(c1[R])-c1ccccc1OC3CCC2</smiles>

$50 \mathrm{c}$

Scheme 11

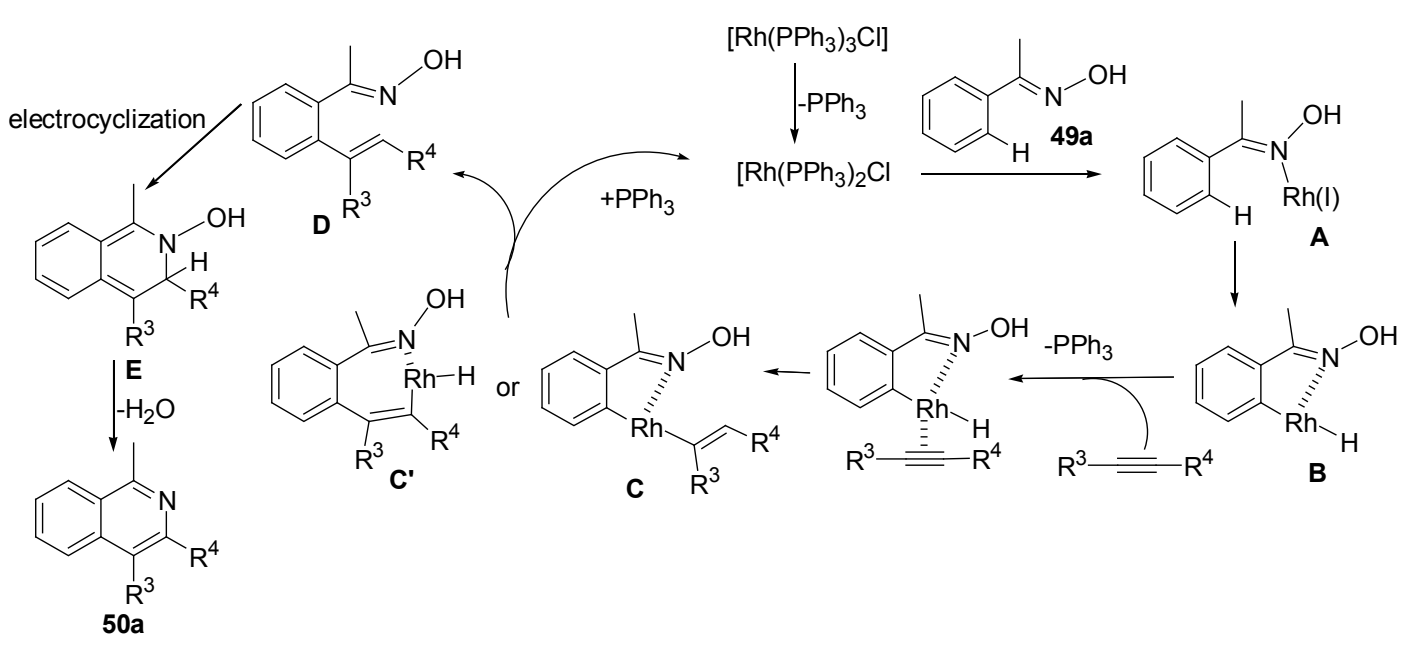

Scheme 12 


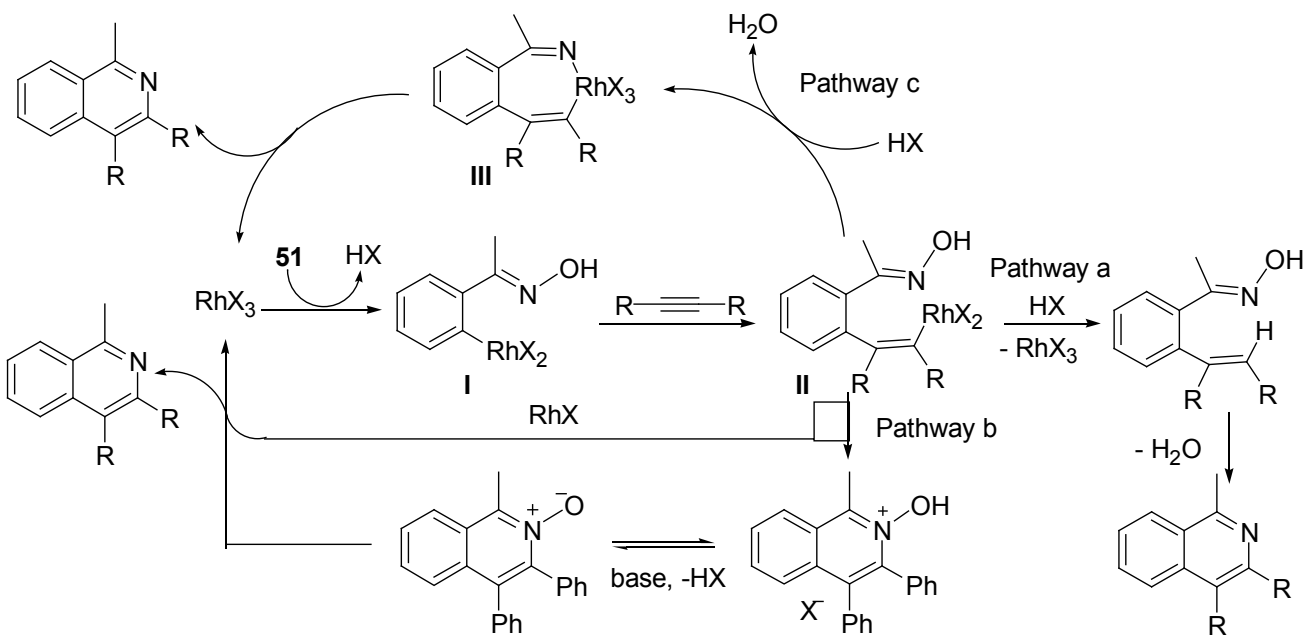

Scheme 13

2010 年, Chiba 等 ${ }^{[31]}$ 将 $\left[\mathrm{Cp}^{*} \mathrm{RhCl}_{2}\right]_{2}-\mathrm{NaOAc}$ 催化体 系应用于肟酯化合物和炔烃的反应, 得到了较好的结果 (Eq. 25). 该反应在中性氧化还原体系里, 以 $\mathrm{CH}_{3} \mathrm{OH}$ 为 溶剂, 反应温度为 $60{ }^{\circ} \mathrm{C}$, 顺利得到了异喹啉类化合物 52. 此反应的特点是肜酯化合物中的含氮定位基团可以 作为一种良好的内部氧化剂作用于催化循环, 避免了以 往该类反应中的常用外加养化剂[32].

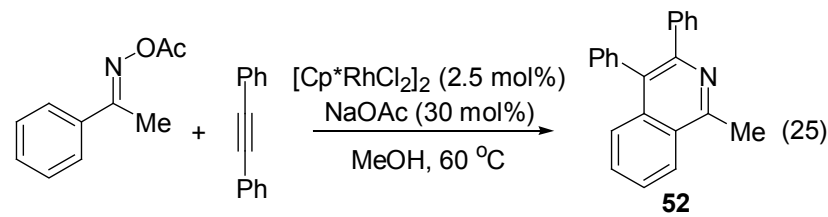

在此研究基础上, 该课题组 ${ }^{[33]}$ 还报道了使用 $\mathrm{Cu}(\mathrm{OAc})_{2}-\left[\mathrm{Cp}^{*} \mathrm{RhCl}_{2}\right]_{2}$ 双金属催化体系, 以 $\mathrm{DMF}$ 为溶 剂, $60{ }^{\circ} \mathrm{C}$ 条件下用芳基酮肜酯与炔烃合成杂环化合物 的新方法(Eq. 26). 通过这种方法, 无论顺式和反式的肪 酯化合物都能得到很好的结果, 甚至含有吲哚、苯并呋 喃、呋喃、吡咯、噻吩基的酮肜化合物反应也都得到较 高的产率. 当用 $\mathrm{Cu}(\mathrm{OAc})_{2}-\left[\mathrm{Cp}^{*} \mathrm{RhCl}_{2}\right]_{2}$ 催化体系代替 $\left[\mathrm{Cp} * \mathrm{RhCl}_{2}\right]_{2}-\mathrm{NaOAc}$ 催化体系时, 可显著提高部分产物 的收率，如化合物 $\mathbf{5 5}$ 的产率可从 $42 \%$ 提高到 $82 \%$.

最近, Chiba 等 ${ }^{[34]}$ 对该反应体系作了进一步优化, 使用 $\left[\mathrm{Cp}^{*} \mathrm{RhCl}_{2}\right]_{2}-\mathrm{CsOPiv}$ 作催化剂, 以 $\mathrm{CH}_{3} \mathrm{OH}$ 作溶剂, 空气为氧化剂, $60{ }^{\circ} \mathrm{C}$ 条件下用 $\alpha, \beta$-不饱和酮肜 61 与炔 顺利合成了吡啶类化合物 62 (Eq. 27).

与此同时, Rovis 等 ${ }^{[35]}$ 也对该类反应进行了深入研 究, 使用 $\left[\mathrm{Cp}^{*} \mathrm{RhCl}_{2}\right]_{2}$ 和 $\left[\mathrm{Cp}^{t} \mathrm{RhCl}_{2}\right]_{2}$ 作催化剂, 以三氟乙 醇(TFE)作溶剂, 在过量的 $\mathrm{K}_{2} \mathrm{CO}_{3}$ 存在下实现了肜类化 合物与非对称炔烃的反应(Eqs. 28,29). 该反应条件温 和, 催化剂简单易制备, 为合成多取代吡啶和异喹啉类 化合物提供了一条新的合成途径.<smiles>[R]c1nc(-c2ccccc2)c(-c2ccccc2)c2ccccc12</smiles><smiles>CC(C)(C)c1nc(-c2ccccc2)c(-c2ccccc2)c2ccccc12</smiles>

$5399 \%$<smiles>c1ccc(-c2nc(-c3ccccc3)c3ccccc3c2-c2ccccc2)cc1</smiles>

$5478 \%$<smiles>Cc1nc(-c2ccccc2)c(-c2ccccc2)c2c1c1ccccc1n2C(=O)OC(C)(C)C</smiles>

$5582 \%(42 \%)$

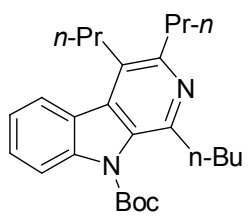

$5691 \%$<smiles>CCCCc1c(C)nc(C)c2cc(CCC)nc(C)c12</smiles>

$5786 \%$<smiles>CCCc1nc(C)c2occc2c1C(=O)O</smiles>

$5891 \%$

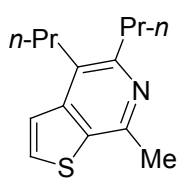

$5991 \%$

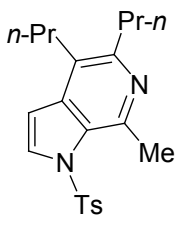

$6091 \%$

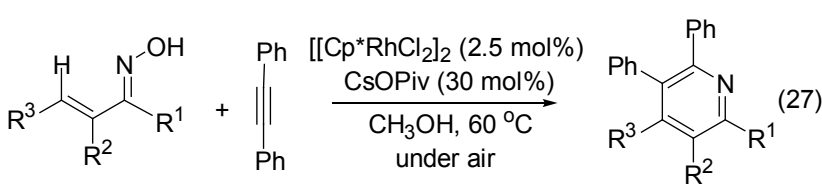

61
62

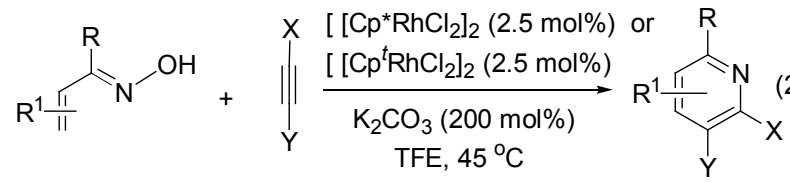




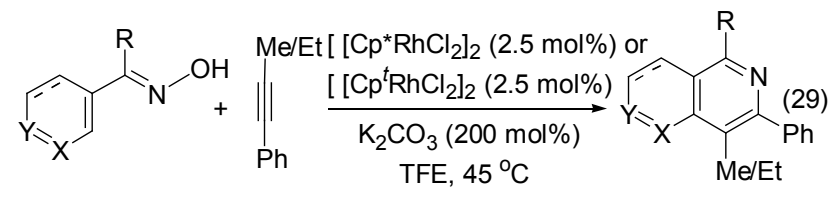

根据实验结果和相关文献报道 ${ }^{[32 \mathrm{~b}, 32 \mathrm{~d}]}$, 他们对反应 的机理进行了研究. 他们认为该反应可能的机理是: 首 先 $\alpha, \beta$-不饱和醛肜与 $\mathrm{Rh}$ 原子中心配位形成络合物 $\mathbf{A}$, 络 合物 $\mathbf{A}$ 在碱的作用下继而发生转化次数限制的 $\mathrm{C}-\mathrm{H}$ 活化反应形成新的五元环络合物 $\mathbf{B}$, 接着插入炔烃得到 七元环金属络合物 $\mathbf{C}$, 最后还原消除即得目标产物, 并 释放出 $\mathrm{OH}^{-}$(Scheme 14).

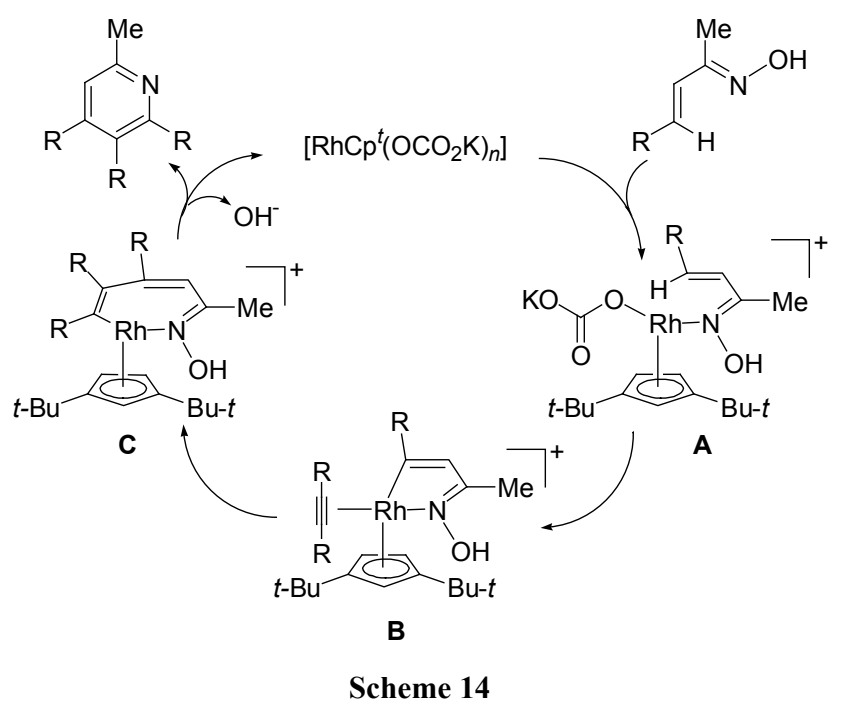

\section{5 结论和展望}

综上所述, 肟类化合物正越来越广泛地被应用于天 然产物或生物活性化合物的合成中 ${ }^{[36]}$. 尽管在过去的 十几年里, 金属催化的肜类化合物反应研究已取得一些 进展, 但是许多方法仍然有待进一步提高, 如催化剂昂 贵, 反应效率差, 对底物结构有局限性等, 因此发展更 为有效的肜的新反应具有重要的意义. 我们相信新的进 展和应用还会不断涌现, 过渡金属催化的肜类化合物反 应将会在有机合成以及相关研究领域占有越来越重要 的地位.

\section{References}

[1] (a) Boar, R. B.; McGhie, J. F.; Robinson, M.; Barton, D. H. R.; Horwell, D. C.; Stick, R. V. J. Chem. Soc., Perkin Trans. 1 1975, 1237.

(b) Boar, R. B.; McGhie, J. F.; Robinson, M.; Barton, D. H. R. J. Chem. Soc., Perkin Trans. 1 1975, 1242.

[2] Barton, D. H. R.; Zard, S. Z. J. Chem. Soc., Perkin Trans. 1 1985, 2191.

[3] Burk, M. J.; Casy, G.; Johnson, N. B. J. Org. Chem. 1998, 63, 6084.
[4] Zhu, G.; Casalnuovo, A. L.; Zhang, X. J. Org. Chem. 1998, 63, 8100.

[5] Zhao, H.; Vandenbossche, C. P.; Koenig, S. G.; Singh, S. P.; Bakale, R. P. Org. Lett. 2008, 10, 505.

[6] Guan, Z.-H.; Huang, K.; Yu, S.; Zhang, X. Org. Lett. 2009, 11, 481.

[7] Tang, W.; Capacci, A.; Sarvestani, M.; Wei, X.; Yee, N. K.; Senanayake, C. H. J. Org. Chem. 2009, 74, 9528.

[8] Guan, Z.-H.; Zhang, Z.-Y.; Ren, Z.-H.; Wang, Y.-Y.; Zhang, X. J. Org. Chem. 2011, 76, 339.

[9] Coffen, D. L.; Schaer, B.; Bizzarro, F. T.; Chung, J. B. J. Org. Chem. 1984, 49, 296.

[10] Prithwiraj, D.; Nonappa; Pandurangan, K.; Maitra, U.; Wailes, S. Org. Lett. 2007, 9, 2767.

[11] Udd, S.; Jokela, R.; Franzén, R.; Tois, J. Tetrahedron Lett. 2006, $47,8247$.

[12] Maimone, T. J.; Buchwald, S. L. J. Am. Chem. Soc. 2010, 132, 9990.

[13] Liu, S.; Yu, Y.; Liebeskind, L. S. Org. Lett. 2007, 9, 1947.

[14] Liu, S.; Liebeskind, L. S. J. Am. Chem. Soc. 2008, 130, 6918.

[15] Feng, X.-H.; Zhang, G.-Z.; Chen, C.-Q.; Yang, M.-Y.; Xu, X.-Y.; Huang, G.-S. Synth. Commun. 2009, 39, 1768.

[16] Ali, A.; Meyer, A. G.; Tuck, K. L. Synlett 2009, 955.

[17] Wang, L.; Huang, C.; Cai, C. Catal. Commun. 2010, 532.

[18] Ren, Z.-H.; Zhang, Z.-Y.; Yang, B.-Q.; Wang, Y.-Y.; Guan, Z.-H. Org. Let. 2011, 13, 5394.

[19] Tsutsui, H.; Narasaka, K. Chem. Lett. 1999, 45.

[20] Tsutsui, H.; Narasaka, K. Chem. Lett. 2001, 526.

[21] Zaman, S.; Kitamura, M.; Abell, A. D. Org. Lett. 2005, 7, 609.

[22] Kamijo, S.; Yamamoto, Y. Chem.-Asian J. 2007, 2, 568.

[23] (a) Narasaka, K.; Kitamura, M. Eur. J. Org. Chem. 2005, 7, 4505. (b) Narasaka, K. Pure Appl. Chem. 2002, 74, 143.

(c) Kitamura, M.; Zaman, S.; Narasaka, K. Synlett 2001, 974.

[24] Tan, Y.; Hartwig, J. F. J. Am. Chem. Soc. 2010, 132, 3676.

[25] Chiba, S.; Zhang, L.; Sanjaya, S.; Ang, G. Y. Tetrahedron 2010 , 66, 5692.

[26] Faulkner, A.; Bower, J. F. Angew. Chem., Int. Ed. 2012, 51, 1675.

[27] Parthasarathy, K.; Jeganmohan, M.; Cheng, C.-H. Org. Lett. 2008, 8,325 .

[28] Parthasarathy, K.; Cheng, C.-H. J. Org. Chem. 2009, 74, 9359.

[29] Zhang, X.; Chen, D.; Zhao, M.; Zhao, J.; Jia, A.; Li, X. Adv. Synth. Catal. 2011, 353, 719.

[30] Martin, R. M.; Bergman, R. G.; Ellman, J. A.; J. Org. Chem. 2012, $77,2501$.

[31] Too, P. C.; Wang, Y.-F.; Chiba, S. Org. Lett. 2010, 12, 5688.

[32] (a) Patureau, F. W.; Glorius, F. Angew. Chem., Int. Ed. 2011, 50, 1977.

(b) Guimond, N.; Gorelsky, S. I.; Fagnou, K. J. Am. Chem. Soc. 2011, 133, 6449.

(c) Rakshit, S.; Grohmann, C.; Besset, T.; Glorius, F. J. Am. Chem. Soc. 2011, 133, 2350.

(d) Guimond, N.; Gouliaras, C.; Fagnou, K. J. Am. Chem. Soc. 2010, 132, 6908.

[33] Too, P. C.; Chua, S. H.; Wong, S. H.; Chiba, S. J. Org. Chem. 2011, 76, 6159.

[34] Too, P. C.; Noji, T.; Lim, Y. J.; Li, X.; Chiba, S. Synlett 2011, 2789.

[35] Hyster, T. K.; Rovis, T. Chem. Commun. 2011, 47, 11846.

[36] (a) Sukhorukov, A. Y.; Ioffe, S. L. Chem. Rev. 2011, 111, 5004.

(b) Alonso, D. A.; Nájera, C. Chem. Soc. Rev. 2010, 39, 2891.

(c) Song, G.; Wang, F.; Li, X. Chem. Soc. Rev. 2012, 41, 3651.

(d) Chinnagolla, R. K.; Pimparkar, S.; Jeganmohan, M. Org. Lett. 2012, 14, 3032 .

(Zhao, X.) 